Revista Digital Año 6. Nº 6 - Año 2015. --pág. 1-65

ISSN 1853-1393

Resistencia. Chaco. Argentina - 2015

\title{
LA FORMACIÓN DE FORMADORES: UN CAMPO A DESCUBRIR DESDE EL PROPIO TRAYECTO PROFESIONAL
}

\section{THE FORMERS FORMATION: A FIELD TO BE DISCOVERED FROM ITS PROFESSIONAL SECTION}

Mg. María Cristina, Alonso ${ }^{1}$ - Mg. Silvia Estela, Ormaechea ${ }^{2}$

Fecha de recepción: 04-06-2015

Fecha de aceptación y versión final: 04-08-2015

Resumen: Al incursionar -desde mediados de la década del 90- en el campo de la formación de formadores, surgió la necesidad de clarificar nuestro posicionamiento epistemológico para su abordaje, lo cual significó una toma en consciencia de sus fundamentos. Así se recuperó lo histórico, lo social, lo cultural y lo subjetivo, como dimensiones inherentes a la complejidad del campo de la formación de formadores.

Desde esta decisión, se seleccionaron tres líneas teóricas - entre otras- que nos aproximan a la comprensión del campo de la formación de formadores: la relación entre epistemología y formación, las ciencias de la educación y el campo de la formación de formadores y la problemática de la formación desde las miradas psicoanalítica, psicosocial y sociológica-culturalista. Para finalizar, utilizamos como dispositivo de objetivación de lo que se sostiene, el análisis de dos momentos singulares de un grupo durante su trayecto de formación profesional posgraduada.

Palabras claves: formación de formadores-trayecto de formación-grupo de formación-análisis, interpretación y comprensión

Summary: Going into - from half of the 90 decade - in the field of formers formation, appeared the neccessity to clarify our epistemological placement to enter upon, that means be conscious of its basis. In thisway the historical, social, cultural and subjective was recovered, as inherent dimensions to complex field in the formers formation. From this decision, three theoretic lines were selected -among others- that put ourselves close to understand the field of formers formation; the relation between epistemology and formation, the sciences in education and the field of formers formation and the problematical of formation from the psychoanalitical point of view, psychosocial and sociological - cultural. To finish with these, we use as an objectiv device of what it is support, the analysis of two singular moments of a group during their way to professional posgraduate formation.

Keys words: formers formation - formation sector - formation group -analysis, interpretation and comprehension

1 Especialista y Mgter en Formación de Formadores (UBA). Docente Titular Ordinaria, dedicación exclusiva, jubilada desde agosto de 2014, en las cátedras de Didáctica II y Grupos e Instituciones Educativas del Dpto. de Ciencias de la Educación. Actualmente Directora de la Carrera de Posgrado: Especialización en Análisis de la Educación, sus organizaciones y sus prácticas. Facultad de Humanidades -UNNE-. Correo electrónico: macristialonso@yahoo.com.ar

2 Especialista y Mgter en Formación de Formadores (UBA). Profesora Titular Ordinaria, dedicación exclusiva, en la cátedra Práctica de la enseñanza en instituciones educativas del Dpto. de Ciencias de la Educación. Facultad de Humanidades -UNNE-Actualmente Directora de la Carrera de Especialización en Docencia y Gestión Universitaria con orientación en Ciencias de la Salud. Facultad de OdontologíaUNNE. Correo electrónico: seormaechea@gmail.com 


\section{Introducción}

Al incursionar -desde mediados de la década del 90- en el campo de la formación de formadores, surgió la necesidad de clarificar nuestro posicionamiento epistemológico para su abordaje, lo cual significó una toma en consciencia de sus fundamentos. En vista a una decisión epistemológica, tres representantes de la epistemología contemporánea fueron sustantivos para tal decisión y consecuentemente- para la selección de modelos teóricos y sus derivaciones metodológicas: Thomas S. Kuhn, Edgar Morin y Fritjol Capra. El primero, por cuanto revolucionó con su tesis historicista respecto del fenómeno de la ciencia y con su orientación más sociológica respecto a la noción de paradigma. El segundo, por cuanto es uno de los representantes del paradigma del pensamiento complejo que encierra una posición dialógica culturalmente inscripta. El tercero, por cuanto generaliza la noción de paradigma científico elaborado por Thomas Kuhn al ámbito de lo social y habla entonces de paradigma social.

Fue a partir de estas tres líneas que las dimensiones de lo histórico, lo social, lo cultural y lo subjetivo se recobraron de manera entrelazada para el reconocimiento de la complejidad de lo real; donde se interrelacionan e interaccionan esas dimensiones en un movimiento recursivo- y representan el punto de apoyo desde donde se pretende pensar, analizar y comprender el fenómeno de la formación.

Se asume así como necesario, tener en cuenta distintas miradas y componer una descripción compleja de las interdependencias entre los mundos simbólico y fenomenológico para interpretar el campo de la formación de formadores sin hacer reduccionismos ni simplificaciones; es decir una lectura plural del entretejido de las múltiple dimensiones considerando la multirreferencialidad de comprensión que nos acerca a la escucha clínica para familiarizarnos con lo simbólico del discurso como lo puesto en acción espontáneamente, la multireferencialidad interpretativa de los datos y la multireferencialidad explicativa que tiene como propósito la producción de saber.

En esta línea es impensable la imparcialidad de los sujetos implicados en el campo en tanto son sujetos indisociables de la cultura y de la sociedad a la que pertenecen y todo lo que pretenden conocer no puede aislarse de su entorno y ni de sí mismos como si fueran objetos inertes. Es decir entonces que los sujetos se convierten en objeto de conocimiento al mismo tiempo que siguen siendo sujetos y la realidad a conocer -siempre heterogénea, multidimensional, compleja- objetiva y subjetiva a la vez. Asimismo son sujetos que escuchan el transitar subterráneo del pensamiento, son individuos-sujetos pulsional y afectivo: los deseos, los temores, las fantasías, lo imaginario se infiltran en las ideas, concepciones y visiones sobre la realidad de tal manera que es imposible pensar que dichas ideas concepciones o visiones sean puras en el sentido de no estar contaminadas por ese sujeto. Al respecto A. Green nos dice: es un sujeto en dos lugares (sujeto de la consciencia y sujeto del inconsciente) lo que nos está hablando de la percusión subjetiva del objeto de conocimiento, de la otra polaridad subjetiva (1991:179)." Esto implica no desinvestir al sujeto, no desubjetivarlo en nombre de la objetivación. Por el contrario, lo importante es tratar o posibilitar un conocimiento objetivable de la subjetividad, de esta manera se abandona la ilusión de ser un externo a lo que se conoce y se asume el verse incluido. Por eso la reflexibilidad de la que nos habla Morin, por eso la autoreflexión de la que nos habla J. Ardoino, en definitiva: por eso la necesidad de considerar el fenómeno de la implicación que tiene que ver con el orden de lo replegado sobre sí mismo y que debe ser comprendida desde una aproximación clínica. El proceso de implicación queda así al descubierto 
Este fue el punto de partida para pensar las ciencias de la educación y el campo de la formación de formadores que se desarrolla en el segundo apartado de este artículo, y desde allí incursionar en diferentes miradas teóricas para el análisis de dos momentos singulares en un grupo de formación durante su trayecto de formación profesional que se exponen en tercero y cuarto, respectivamente.

\section{De las ciencias de la educación al campo de la formación de formadores.}

La educación, siguiendo a J. Ardonio, tiene por meta que los sujetos -destinatarios de aquélla- conquisten los medios psíquicos para su autorización -entendiendo a ésta como el hecho del autoriii, de aquél que crea, de quien logra situarse, él mismo, en el origen, en la fuente de su propio devenir (Ardoino, J.; 1998:73)- así como la aceptación de su alteración $n^{i v}$ y de la aprehensión de la alteridad". De esta manera, los sujetos, a través de su praxis (interior y colectiva) entran en la vida, en la historia y en la sociedad, haciéndose más autónomos, sin perder de vista las relaciones de interdependencia, los lazos sociales que los unen a la comunidad, siempre y cuando den sentido a sus vivencias, a sus actos, al Otro en él, a los otros en su ambiente, es decir a su relación con el mundo.

Por parte de los educadores requiere entonces que consideren al sujeto no sólo por su intencionalidad consciente sino también por su intencionalidad inconsciente, por sus relaciones intra e intersubjetivas, desde una perspectiva clínica y desde diferentes miradas: psicológica, psicosociológica, sociológica, pedagógica, políticas -entre otras(Ardoino, J. 1998).

Esta perspectiva clínica, compleja multireferencial y multidimensional, implica una nueva cosmovisión sobre las Ciencias de la Educación que rompe con el discurso de carácter prescriptivo sobre las decisiones respecto de la elección de los fines, objetivos, medios y estrategias dentro del orden de las prácticas educativas. Es entonces, en términos de Ferry (1990) un discurso que se desenvuelve en un espacio intermedio entre la práctica y la ciencia; que focaliza su atención en la antinomia de los procesos de enseñanza-aprendizaje los cuales fueron al mismo tiempo su conquista y su debilidad. Su conquista, porque permitió discriminar que el problema central de la educación es una relación y su debilidad al generar la antinomia irreductible de lo social y lo individual, de esta manera funda el proceso formativo en antinomias: coerción - libertad, modelización - autonomía, conformismo - emancipación, represión revuelta, conflicto - adhesión, enseñanza - aprendizaje, transmisión - apropiación.

Podemos pensar a las Ciencias de la Educación desde otro paradigma en el cual se las reconoce como un campo complejo donde se admite lo plural que corresponde a las ciencias humanas y sociales, lo transdiciplinar, lo multireferencial, lo que no significa una maraña de saberes, sino un campo heterogéneo de saberes que se articulan.

En esta perspectiva, siguiendo a J. Ardoino (1997; 2001), se asume que ese campo está conformado justamente por un mestizaje, por lo plural, en el cual la multiplicidad de lecturas por las diferentes disciplinas que lo conforman, se oponen, se relacionan, son inconciliables e irreductibles pero a la vez se articulan y en esto consiste justamente su carácter heterogéneo y a la vez homogeneizante en la medida esperada, respetando la imposibilidad de unificar. Esta es la especificidad de las Ciencias de la Educación. A ese campo podemos aproximarnos a su conocimiento siempre y cuando, por un lado, intentemos dar un lugar al sentido y a las significaciones, es decir, intentemos comprenderlo por eso podemos llamarlo también el campo de las ciencias de la comprensión y, por el otro, asumamos que por el hecho mismo de ser un campo en donde se juegan intersubjetividades no puede dejarse de 
lado el análisis de la implicación y por ello podemos llamarlo también el campo de las ciencias de la implicación cuya dimensión fundamental es la temporalidad por cuanto aquello que es implicado o replegado se desarrolla en el tiempo de la duración.

Desde este encuadre el análisis y comprensión de la formación de formadores, es intentar descubrir e interpretar el entretejido de constituyentes heterogéneos de toda situación singular en este campo: el formador y el sujeto en formación (cada uno con su historia personal y profesional), sus relaciones vinculares y de implicación (en donde se confronta lo implícito y lo explícito de los deseos y de las expectativas), las prácticas del formador de formadores y el análisis de dichas prácticas, la situación de formación o dispositivo analizador en tanto continente afectivo; así como lo grupal, el espacio organizacional, institucional, social con sus múltiples atravesamientos -que contienen, configuran su encuadre- y a la vez atraviesan a cada uno de aquellos constituyentes y a su relación entre ellos.

Lo esencial de la formación y la educación es precisamente formar individuos que pueden pensar por cuenta propia, capaces de actuar pensando en las consecuencias de su actuar y capaces de interrogarse y cuestionarse cuando su acción tuvo efectos distintos a los que esperaba. Todo esto implica que el proceso pedagógico en sí mismo debe tener una dinámica vital sin la cual no hay formación. También implica que a través de este proceso lo que se juega para todos nosotros es nuestra capacidad de sublimación, es decir, el placer de pensar y el placer de pensar con otros de manera compartida (Enriquez, E., 2000).

Por eso -y para finalizar este punto- es importante insistir en los aspectos relacionales tanto visibles como inconscientes e imaginarios que implican al conjunto de la personalidad situados en el contexto de un grupo -en una relación de co-formación-, de una institución en un contexto sociohistórico y político que condicionan la manera en que las relaciones pueden llegar a establecerse.

\section{Distintas lecturas para el análisis de la formación de formadores}

\section{Una mirada psicoanalítica y psicosocial}

Parafraseando a Etchegoyen, el psicoanálisis es una forma especial de psicoterapia y es mérito de S. Freud de llevarla a nivel científico con la introducción del psicoanálisis. Esta orientación de la práctica terapéutica y su teoría le significó a Freud romper obstáculos epistemológicos, teniendo en cuenta el momento histórico del desarrollo de la ciencia: crisis en las ciencias de la naturaleza, los nuevos aportes de la antropología, de la ciencia positiva, el desarrollo de la sociología, entre otros ${ }^{\text {vi }}$. No fue otra cosa que la permanente interrogación desde su práctica clínica, lo que lo llevó al mismo tiempo a la práctica investigativa, a la creación, a la elaboración de su corpus teórico que en líneas generales se caracterizar por una teoría de base (la metapsicología), por sus fines prácticos (fantasía inconsciente) y por un método (el dispositivo).

Al hablar de formación desde esta línea teórica, hay que considerar su dimensión básica que es el reconocimiento de la existencia de fenómenos inconscientes, de preocuparse por los procesos inconscientes, ya sea en una relación, en un sujeto en situación, en el grupo, en las instituciones o en la sociedad si es sociólogo (Blanchard Laville, 1995:25).

En Francia esta perspectiva clínica es utilizada tanto en psicología como en sociología. Actualmente se orientó hacia un enfoque más general: el enfoque clínicovii en ciencias humanas. Esta nueva orientación se funda en el hecho de que los objetos 
de investigación se amplían: del sujeto al sujeto en situación para luego considerar al grupo, a la institución y a la sociedad y, a cada uno, desde una perspectiva histórica.

Para pensar entonces en un proceso de formación de futuros formadores, las perspectivas histórica y situacional-clínica tendrían como condición avanzar en la puesta en relación por un lado, el pasado, el presente y expectativas futuras configurando un tiempo en movimiento que incorpora las vivencias afectivas y los componentes inconscientes distorsionantes (Corvalán de Mezzano, 1996). Por el otro, integrar las relaciones del sujeto en situación en una estructura espacio-temporal personal e institucional donde está presente lo vivido con sus componentes individuales y colectivos, psicológicos y sociopolíticos, así como lo explícito y lo implícito (simbólico e inconsciente).

Es en la situación singular en la que se re-crea desde nuevas perspectivas, el camino de la formación, en la que los sujetos implicados son autores de la situación. De lo que trata es que los que participan en una situación de formación puedan experienciar los efectos del inconsciente: proyecciones e identificaciones, actualización de deseos, defensas, conflictos. Implica una movilización, en los sujetos de los procesos psíquicos primarios y secundarios a través de situaciones en la que los procesos psíquicos se encuentran personalmente implicados, movilizados y esclarecidos en su dinámica.

La formación -este núcleo sustantivo- entonces, alude a algo más que a la posibilidad de apropiarse de un saber transmisible por otro, alude también a un saber sobre el propio saber del sujeto que es intransmisible y que sólo es posible comprenderlo e interpretarlo a partir de las posibilidades que brinda la situación de formación que inevitablemente conlleva a peligrosos niveles de de-formación y conformación (Alonso, M. C.; 2006).

Es un trabajo sobre sí mismo, es una acción reflexiva sobre sí mismo, sobre las prácticas profesionales de cada uno y en relación con lo que sucede en el interjuego entre el formador y el formando -es una aventura en común- dentro de un espacio institucional. Esa acción reflexiva requiere de la capacidad de análisis tanto desde el nivel consciente como desde el inconsciente en función de la/las situación/es singular/es. Es el tiempo y espacio necesarios para la elaboración de lo que se vive como pérdida, como ruptura, como de-formación. Generar un espacio transicional (Winnicott; 1977) es también generar un tiempo que se presenta como tiempo vivido -experimentado de manera subjetiva e intersubjetiva que facilita la recusividad necesaria para el trabajo sobre sí mismo-, y que resulta ineluctable para que las carencias puedan ser señaladas y oídas, para enfrentar la incertidumbre, para soportar la frustración y convivir con el duelo del pasaje de una forma a otra que moviliza el ideal de alcanzar una perfección sin mezcla, sin falla y sin división. Pasaje que también conlleva este riego terrorífico: el de ser de-formado, destruido. (...) uno se forma deformándose" (Kaës, 1979:46).

Es un diálogo entre personas por lo tanto implica siempre una perspectiva relacional entre formador y formando, es un diálogo entre inconscientes, es estar atentos a...lo que tiene que ver con el orden del deseo, de lo afectivo, del odio, del miedo, de la culpa de la angustia y también de lo que surge del inconsciente y del orden de lo que tiene que ver con lo intelectual, es decir, el funcionamiento de la inteligencia (Filloux; 1996:25).

Es un proceso de reconstrucción y ejercicio de apropiación del propio acto, una toma de conciencia de su lugar en el espacio social en el sentido de G. Mendel; es el encuentro entre dos saberes y de dos saberes que no son simétricos (el del formador y el del formando) que requiere de su apropiación, es necesario (...) que haga de ella un tema personal a través de su personalidad y su experiencia (1996:113). 
Es un camino desde donde uno no deja de preguntarse sobre el propio proceso de formación, de intentar comprenderlo y desde allí descubrir:

- que el descubrir es la única manera de conocer,

- que siempre está presente el diálogo entre inconscientes,

- que todo proceso de formación implica una deformación y por lo tanto aprender a soportar la frustración de convivir en muchos momentos con el duelo del pasaje de una forma a otra,

- que en cada pregunta que el formador y el sujeto en formación se hace está el otro y de allí la importancia de interrogarse desde una perspectiva ética acerca del sentido de la necesidad de poder de unos sobre otros,

- que la creación de espacios como interlocutorios válidos para el grupo y para cada uno de sus miembros es una vía para el movimiento de apropiación del acto de reflexión personal y colectiva, del acto de formación.

Todo la expuesto nos muestra la importancia de desarrollar la capacidad de análisis y entrenamiento para la observación; la escucha; la interpretación de actitudes, de las interacciones y de las relaciones de poder; para cuestionar, interpretar y comprender las situaciones in vivo o en la evocación que se hace dentro del seno de un grupo de formación (Ferry; 1990).

\section{Una mirada desde lo social}

La pregunta que nos orientó fue ¿Qué teorías podrían contribuir a una nueva interrogación, a una nueva mirada al objeto en análisis? Frente a estas preguntas nos inclinamos, diríamos "casi"viii arbitrariamente, por centrarnos en el pensamiento teórico crítico de la década del $70\left(\mathrm{~S}\right.$. XX) ${ }^{\text {ix }}$, dentro de éste en la teoría de la reproducción de Pierre Bourdieu. En primer lugar no podemos dejar de mencionar, haciendo eco de sus postulados, las teorías que de una u otra manera influyeron en la construcción de su pensamiento tales como las de K. Marx, E. Durkheim, M. Weber, A. Schutz, G. Bachelard, M. Focault; de esta manera ya sea por diferencia, contradicción, similitud, dan el marco para la interpretación de su pensamiento. Su teoría social representa una crítica a las instituciones y convenciones que tiene por objetivo mantener el "status quo" y las desigualdades sociales, es decir, es un exponente crítico de los poderes instituidos; representa una crítica al papel cultural de la enseñanza como reproductora de la distribución jerárquica del capital cultura $^{x}$, una crítica a la violencia simbólica ${ }^{x i}$ que desde el poder simbólico se ejerce para mantener las relaciones jerárquicas de clase y por lo tanto de desigualdades. Desde este escenario la educación es el poder arbitrario que impone la cultura de clase dominante (arbitrariedad cultural).

Si bien Bouirdieu no centró su mirada en la formación, desde su encuadre y propuesta es posible que el formador y el sujeto en formación encuentren nuevas formas a través de los procesos planteados por este sociólogo: reflexividad y autoanálisis como vía para explicitar las categorías de percepción y de apreciación (disposiciones) que estructuran desde adentro las acciones y representaciones de aquéllos. Se podría pensar entonces que ese retorno sobre sí mismo, esa posibilidad de encontrar nuevas formas, implica procesos que Bourdieu denomina de reflexividad y autoanálisis a través del cual sería posible: (a) explicitar las categorías de percepción y de apreciación (disposiciones) que estructuran desde adentro las acciones y 
representaciones del formador y de los sujetos en formación, o en otras palabras su toma de posición en el campo; (b) descubrir/revelar en el devenir personal y profesional las disposiciones duraderas y transferibles, estructuras estructuradas predispuestas para funcionar como estructuras estructurantes; (c) interrogarse sobre el proceso de interiorización de las estructuras externas objetivas (en el formador como en los sujetos en formación); (d) analizar reflexivamente los condicionamientos objetivos de las propias prácticas de formación y así modificar las percepciones y representaciones de los condicionantes externos de esas prácticas y de ellas mismas, y elaborar diferentes estrategias de acción; sería posible el análisis sobre la codificación de las prácticas; (e) reflexionar sobre las posibilidades de aprehender la lógica, que ponen en marcha los formadores y los sujetos en formación en tanto agentes sociales, que producen su práctica en un tiempo y contexto determinado; (f) revelar las estructuras más profundamente ocultas en el habitus individual y en el habitus de clase, en la matriz simbólica de las actividades prácticas, conductas, pensamientos, sentimientos, juicios y los mecanismos que tienden a asegurar su reproducción o transformación; sería preguntarse sobre los valores y principios que regulan el espacio social de pertenencia y la posición en ese espacio; sería el análisis del colectivo individualizado por el rodeo de la incorporación, o del individuo biológico "colectivizado".

Así la reflexibilidad y el autoanálisis que nos plantea Bourdieu, serían una labor de objetivación del sujeto objetivante que debe ser realizada por todos los comprometidos en el campo de la formación de formadores. Cuando se alude al trabajo sobre sí mismo, al retorno sobre sí mismo, desde esta mirada sería la reflexibilidad sobre el trayecto de formación en tanto realidad social, del formador y de los sujetos en formación, en el sistema de relaciones de clase de los hechos (reales o posibles) del que forma parte sociológicamente.

Al trayecto singular se lo comprende en tanto serie de posiciones sucesivas ocupadas por un mismo agente en un espacio sometido a incesantes transformaciones, inserto en el campo ideológico del que participa y que a la vez muestra la posición que ocupa en el campo profesional al que pertenece así como en el campo de poder y su posición en relación con otros campos; supone reconocer las ataduras, las raíces de las estructuras de percepción, de representación en un campo de fuerzas que comandan las prácticas y las ideologías. Es decir, en tanto se haya podido constituir los estados sucesivos del campo en el cual se ha desarrollado el conjunto de relaciones objetivas que han unido el agente considerado -al menos en un cierto número de estados pertinentes- al conjunto de los otros agentes incluidos en el mismo campo y confrontados a las mismas posibilidades (Bourdieu, P. 1986: 3).

Por lo tanto, las trayectorias personales y profesionales definen la forma de inserción en un espacio laboral construido socialmente a la vez que éste le impone exigencias. Las historias de formadores y formandos restituyen la configuración real de la carrera personal y profesional que indica valores y normas que se activan en momentos claves de la vida y contribuyen a explicar los trayectos. El análisis del trayecto, la reflexibilidad sobre el trayecto, produce la posibilidad de reconocer, tanto en la diacronía como en la sincronía temporal y espacial, la reproducción o la transformación construidas que marca el recorrido de las prácticas en los campos sociales así como habitus que singulariza y perfila la identidad social. Cuando se recorre ese camino se puede pensar en la identidad social, como una síntesis, es decir, como la construcción de lo social al identificar las dimensiones psíquicas institucionales, grupales y de ellas las individuales.

Nos dice Wuacquant: 
...entre más tomen consciencia de lo social dentro de ellos mismo, otorgándose un dominio reflexivo de sus categorías de pensamiento y acción, menos probabilidades tendrán de ser actuados por la exterioridad que habita en ellos (1995: 36).

Por lo tanto, la reflexibilidad institucionalizada operaría como mecanismo crítico de formación, diálogo y evaluación.

No se puede, entonces, desterrar las marcas sociales, (comenzando por las "ideas recibidas", lugares comunes donde todo un grupo se reúne para reafirmar su unidad reafirmando sus valores y creencias propias y donde se revelan infaliblemente la posición social de la identificación satisfecha a esta posición de los que la profesan) si lo social no es pensado, si no hay "toma de conciencia" del sistema de disposiciones inconscientes, que es el producto de la interiorización de las estructuras objetivas [habitus] y que, en tanto que lugar geométrico de los determinismos objetivos y de una determinación del porvenir objetivo y de las esperanzas subjetivas, tiende a producir prácticas $y$, por ello, carreras objetivamente ajustadas a las estructuras objetivas. (Bourdieu, P. 2002: 41-42)

El formador y la situación de formación, en tanto mediadores, representarían al analista y al analizador que posibilitarían la reflexión de los sujetos en formación sobre las condiciones sociales y los condicionamientos de los que son producto su trayecto, su formación, sus experiencias profesionales, todo lo que disimula y se revela a la vez en ese diálogo intersubjetivo, la pronunciación y la entonación así como el lenguaje del cuerpo, los silencios, los sobre entendidos, los lapsus (Bourdieu, P. 1999). Pero también el formador, para no oscurecer su mirada, debe analizar sus condicionantes sociales que lo afectan como analista tales como: sus características personales (clase, sexo, etnia), su posición en el microcosmo del campo académico, su compromiso con los problemas de la realidad del sujeto que reclama soluciones prácticas.

Entonces, desde esta resignificación del concepto de formación, a partir de la propuesta de Bourdieu, se presenta el autonálisis, la reflexivilidad, y la mirada del formador como posibilidad para producir transformaciones en el habitus individual y profesional en el campo de la formación de formadores.

\section{Dos momentos singulares en un grupo de formación durante su trayecto de formación profesional}

\section{Un marco de referencia para el análisis}

Interesa ahora recuperar el planteo de dos momentos singulares en un grupo de profesores universitarios -en el que están incluidas las autoras de este artículo- que decidieron iniciar el trayecto de formación profesional en una carrera de especialización que luego continúo en una maestría referidas -ambas- al campo de la formación de formadores.

Transcurría el año 1995, la reforma educativa implementada resultó un tiempo signado por enfrentamientos. No era la primera vez que esto ocurría. En ese entonces y en diferentes momentos de los trayectos profesionales como profesoras en Ciencias de la Educación abocadas a tareas de orientación técnica, gestión institucional -y fundamentalmente, a cargo de cátedras de nivel terciario y universitario- frente a algunas prescripciones y líneas de trabajo provenientes de la entonces Ley Federal de Educación Superior y de algunos documentos del Consejo Federal de Cultura y Educación, encararon modos de responder con criterios que eran duramente resistidos. En ese año, los juegos del poder irrumpieron de una manera peculiar en la 
institución de origen. "El grupo" -así lo denominaremos a partir de aquí- sintió la necesidad de protagonizar decisiones y de fijar criterios singulares sobre cuestiones referidas al cómo abordar los procesos de formación en la universidad. Estaba convencido, que las instituciones debían encarar un mejoramiento cualitativo realizando convenios entre ellas. Las universidades con historia y prestigio académico reconocido, debían ser sensibilizadas para asumir una contribución solidaria en la oferta y desarrollo en formación de post-grado. Otros grupos, por el contrario, centraban la discusión en la organización de cursos a cargo de destacados especialistas. Independientemente de las virtudes de esos especialistas, en el fondo, su selección obedecía a conocimientos individuales que se poseía acerca de ellos o se argumentaba acerca de la experiencia que tenían en tanto organizaban cursos de post-grado en otras instituciones. Por cierto, la batalla se perdió y la institución a la que pertenecía "el grupo", siguió los derroteros que las circunstancias fueron imponiendo a la luz de las relaciones de poder institucional. Sin embargo, la responsabilidad y la firme y sostenida conducta de abocarse al estudio sistemático y continuado, actuó como cinturón protector para "el grupo", de esta manera la derrota se transformó inesperadamente en triunfo: pudo acceder a una institución universitaria que a su criterio reunía las condiciones de idoneidad, calidad con oferta atractiva de saberes deseados y largamente esperados, y ofrecía un modo particular de abordar la problemática de la formación.

Sorteó primero el difícil trance de la inscripción. Nada más molesto que escribir el curriculum vitae y realizar todos aquellos ritos que caracterizan el ingreso a una actividad: fichas de inscripción, certificaciones, notas, entre otras acciones. Además una entrevista individual con la Directora de la Carrera: el fantasma de la entrevista, obraba de manera diferente, constituía un hito insalvable para una, para las otras no revestía un obstáculo invencible. "El grupo" contuvo y pudo avanzar hacia su objetivo.

De su institución de origen lo separaban $1000 \mathrm{~km}$. de distancia, pero, cualidad extraña del deseo, el espacio desapareció y "el grupo" se encaminó hacia allí, a la vuelta de la esquina. Caprichoso juego del deseo, largamente aletargado pero siempre presente: volver, y siempre volver; se encontró en el punto de partida y sin embargo, paradójicamente, también en el punto de llegada.

Y comenzó a estudiar. Un francés seductor, lo atrapó con el arrullo de su voz, y se comenzó a discutir otra vez: la formación, ¿qué es la formación? Discusiones e intercambios con J. Beillerot sobre la relación con el saber, con G. Ferry sobre la pedagogía de la formación, con J. C. Filloux y el diálogo entre inconscientes, con G. Mendel el fenómeno de la autoridad y el acto poder, con C. Blanchar Laville sobre el saber y la relación pedagógico, con N. Mosconi el planteo sobre la diferencia de sexo y la relación con el saber, con J. Ardoino sobre la formación desde la perspectiva de lo complejo; entre otros. Y se instaló al fin Freud y con él la angustia de la legitimidad de su irrupción en la formación de formadores.

\section{Primer momento singular: el grupo y el grupo grande}

Mientras "el grupo" sentía el placer de compartir encuentros en la tarea de formarse, aparecieron cuestiones inherentes a la problemática de la formación que se iniciaba en el grupo grande de la carrera donde se derribaban muros y se generaba discordia. Éste plantea la queja relativa a que no se acepta su participación y la recuperación de sus experiencias profesionales. Cuando en realidad lo que implícitamente se ponía en discusión eran detalles acerca de rutinas propias de situaciones escolarizantes pero no los aspectos sustantivos de la carrera. La respuesta institucional, fue la vuelta al contrato. 
Frente a esta situación, "el grupo" asumió la queja del grupo grande pero no el enfoque. Por esta circunstancia aceptó la respuesta de los docentes de la carrera como propia. Era el contrato pedagógico asumido al comienzo el punto de referencia, que no se quería romper o violar. Es decir, no deseaba que se cuestionaran los límites dados por la bibliografía, las clases, el prestigio profesional de los profesores y los dispositivos grupales ofrecidos.

¿Por qué "el grupo" se mantuvo en esa posición? ¿Qué entendía que la carrera ofrecía? ¿Cómo se ubicó en el proceso de formación?

Las respuestas a estas preguntas lo llevó a plantearse como objeto singular de análisis, la propia situación de formación y como variables de análisis: rasgos, tensiones y necesidad de cambios. Los componentes que interactuaron fueron:

- lo individual $\quad$ - lo colectivo

- lo manifiesto - lo latente

- lo psicológico - lo psicosociológico

- lo subjetivo - lo real

Desde el punto de vista institucional la carrera previó dispositivos de formación que parafraseando a M. Souto (1999) representan un mediador y generan espacios al servicio de la producción, de lo nuevo, del cambio, del desarrollo del sujeto, de la grupalidad y de lo instituyente; un espacio-tiempo en el que está presente lo social, el poder, lo ideológico, lo cultural, el conocimiento, el deseo, en función de la intencionalidad pedagógica de facilitar la formación. Y fue a través de los seminarios, los talleres, las pasantías y, por último, la elaboración de la memoria profesional previstos por la carrera- que se hicieron tangibles para "el grupo" los dispositivos de formación. Tenían la intencionalidad de un trabajo sobre el propio grupo en función de la singularidad de las situaciones por las que se atravesaba, a fin de que el grupo-por un lado- y cada uno de sus miembros individualmente -por otro- desestructuren y estructuren el conocimiento de la realidad y de posibilitar la puesta en escena el deseo del instituyente. El supuesto de base era que las experiencias serían reconstruidas en nuevos niveles por interacción con los marcos teóricos propuestos y por la interacción grupal en los diferentes dispositivos seleccionados a tal efecto. Cada uno en su singularidad aportaban el aparato psicosocial necesario para asegurar la continuidad del ser y la contención para soportar la crisis que conlleva los procesos profundos de transformación.

\section{Segundo momento singular: el sujeto, el grupo, la grupalidad}

En la perspectiva individual de cada miembro de "el grupo" hubieron procesos de reconstrucción: aceptar -en términos de Filloux, J (1996)- que antiguos hábitos pueden transformarse en conocimientos nuevos o luchas contra saberes anteriores. En este punto, se rescatan experiencias a partir de las situaciones inevitables en la intrasubjetividad de la formación: a) ansiedad o angustia, b) refugio en los rituales, c) acciones subterráneas de la transferencia.

El primer momento del trayecto de formación se tradujo en ansiedad o angustia. Esta angustia se la interpretó como: "no fallar a la institución universitaria donde se desarrollaba la carrera (sustituto del padre) para no destruirnos". La ansiedad o angustia surge del juego de los mecanismos de identificación expuestos por Freud, el sujeto descubre en sí un rasgo común con otra persona que no es objeto de sus instintos sexuales. Este proceso de idealización se presenta como ldeal del Yo (profesores) y como un proceso defensivo. 
Esto siguió el camino de una regresión y por consiguiente una réplica de situaciones ya vividas: la situación de escolaridad con controles continuos. La organización de la carrera, tratándose de una formación de adultos, de adultos ya profesionales, no estableció los controles esperados. La angustia resultó ser por no encontrar los dispositivos esperados de acuerdo con la regresión realizada. La escolarización mencionada tuvo el sentido que permitió pasar a los otros aspectos de la intrasubjetividad de la formación: refugio en los rituales y acciones subterráneas de la transferencia.

El deseo de ser intérprete de la propia situación y correlativamente esclarecer los procesos primarios en juego, no se logró sino finalmente gracias a la circulación grupal por un proceso de transferencia donde "el grupo" asume la angustia individual como grupal y demanda analizar (interpretar) no un caso cualquiera, sino la propia situación de formación.

Este es el punto de inflexión en el cual se comenzó a discriminar lo manifiesto y lo latente y, por lo tanto, hacer inteligible el inconsciente. Estamos situados en el momento de la interpretación reparadora (Filloux; 1996) de la angustia tanto a nivel individual como grupal e institucional. Se está dando lugar a la fuerza del "movimiento de apropiación del acto" (del acto-poder) que se "vincula con mutuos actos en curso de ejecución o bien ya ejecutados y que nos remite a nuestra responsabilidad", y esto fue posible por la exigencia colectiva de trabajo para alcanzar el más allá de la autoridad" (Mendel, 1996:71-73). Desde la perspectiva de lo colectivo, tal como lo dice G. Mendel (1996), no se puede negar que existen movimientos colectivos pero al mismo tiempo nada existe en él que no existiese ya en germen en la representación individual.

Al situarse el grupo en el momento de la interpretación reparadora, la relación con el otro en la intersubjetividad creó el espacio transicional (Winnicott, 1977) y desde ese espacio fue posible volver sobre sí (de una conciencia de sí a una conciencia para sí (Filloux; 1996)

\section{¿Qué significó para "el grupo" el proceso antes descripto?}

Las bases fundacionales de la formación de "el grupo", se tambalearon; tuvo que admitir nuevas reglas en el estudio sistemático en una institución con ofertas superiores para funcionar con productividad: producir, saber competir, admitir la incertidumbre. Sus experiencias anterior se la representaba como una situación "fetal" en la que el alimento provenía de los textos de la especialidad y el espacio-continente era la cátedra o el cargo, los cuales no estaban sometidos a discusión y representaban, por lo tanto, la estabilidad y la seguridad. Al aceptar la alteración, la incertidumbre actuó no ya como pérdida de estabilidad sino como "ruptura de la relación inter e intrasubjetiva " (Kaës, 1993:26) y con generatividad, es decir, con productividad y creatividad, por lo tanto con la posibilidad de "...generación de nuevos productos e ideas, incluido un tipo de autogeneración que tiene que ver con un mayor desarrollo de la identidad" (Erikson, 1985:85). A pesar de esa posibilidad cabría aquí la pregunta que Kaës (1979) plantea en su propuesta de análisis transicional "¿Qué le ocurre al sujeto en ese intervalo entre una pérdida segura y una incierta adquisición, en el momento en que todavía no se han establecido nuevos lazos suficientemente seguros y confiables con un 'ambiente' diferente, en el momento en que el espacio psíquico y social necesarios para articular lo antiguo y lo nuevo no está todavía constituido y el tiempo se presenta como suspendido, congelado y neutralizado?" (Kaës y otros; 1979:27). La carrera proveyó "encuadres" (Bleger, Kaës), dispositivos de formación suficientemente contenedores para que se conviertan en el "espacio transicional" necesario para la ruptura y dispuesto a la articulación psicosocial. La 
institución (carrera) permitió delimitar los metasistemas que contuvo a "el grupo", sin los cuales no hubiera podido "depositar la parte psicótica de nuestra personalidad" para poder encontrar la entrada al mundo y permitir la creación -parafraseando a Kaës-, R (1979), ¿Cómo toleró la paradoja? Porque intentó hacer un análisis transicional, "aquello que haciendo posible el análisis escapa a él".

Esos encuadres, además permitió al "el grupo" cuestionarse sobre las marcas sociales (Bourdieu, 2000), que lo posicionaron desde "otro lugar" en la reconstrucción de lo social. Al identificar las dimensiones individuales, grupales e institucionales logra una conciencia del sistema de disposiciones inconscientes para producir transformaciones en el habitus individual y profesional en el campo de la formación de formadores. La reflexibilidad institucionalizada como mecanismo de diálogo y análisis, fue la vía por excelencia para reconocer el sistema de relaciones de clase de los hechos (reales o posibles) del que forma parte sociológicamente.

\section{Reflexiones de el grupo acerca del después}

El camino recorrido ofreció la posibilidad de incluir los parámetros psicoanalíticos, psicosociológicos, sociopsicoanalíticos y sociales -entre otros- para pensar las diferencias entre un enseñante y un formador, y simultánea e ininterrupidamente tomar como caso la historia personal y profesional.

La reconstrucción del trayecto de formación quedó planteada como un proceso cuyo desarrollo y articulación -en los diversos y complejos espacios sociales- está dado por un interjuego psicosocial en el que las "ilusiones" omnipotentes se limitan, en el que los supuestos frenos se liberan y se descubre por fin que las obstrucciones estaban en el grupo. Nunca antes el grupo y cada uno de sus miembros individualmente, se había tomado el tiempo de volverse sobre sí mismo para descubrir las múltiples dimensiones que se necesitan desentrañar para la compresión de la situación de formación.

\section{BIBLIOGRAFİA}

Alonso, M. C. (2006). "Dispositivos de Acompañamiento", en: Revista Nordeste Investigación y Ensayos - 2da. Epoca № 25: pp 3-14.

Alonso, M. C. (2008). "El acompañamiento a practicantes en períodos de formación: una aproximación desde el estado del arte", en Revista Digital del Instituto de Investigaciones en Educación - Facultad de Humanidades de la UNNE. Resistencia. http://hum.unne.edu.ar/revistas/educa/index.htm

Alonso, M. C. (2011). "El posicionamiento epistemológico, metodológico y encuadre teórico de una investigación en curso", en Revista Digital del Instituto de Investigaciones en Educación - Facultad de Humanidades de la UNNE. Resistencia. http://hum.unne.edu.ar/revistas/educa/index.htm

Anzieu, D. (1993). El Grupo y el Inconsciente: Lo Imaginario Grupal. Madrid: Biblioteca Nueva.

(1995). El pensar. Del Yo-piel al Yo-pensante. Madrid: Biblioteca Nueva Anzieu, D.; Houzel, D. y otros. (1987). Las envolturas psíquicas. Buenos Aires: Amorrortur.

Ardoino, J. (1997). "La Implicación". Conferencia en el Centro de Estudios sobre la Universidad. Mimeo. Universidad Autónoma de Madrid.

(1998). Referencias y notas de lectura. Fragmentos de textos, nociones y definiciones: grupos organizaciones e instituciones. Méjico. Universidad lberoamericana. 
(2001). "Para una educación que finalmente se reconozca mestiza (estatutos respectivos de la heterogeneidad y de la impureza a partir de esa óptica)". Comunicación para el coloquio de AFIRSE (Natal-Brasil).

Barbier, R. (1977). La recherche. Action dans 'I institution educative. Segunda Parte: "Aproximación teórica específica" Traducción para la Cátedra Observación y trabajo de campo II (UBA). Mimeo.

Beillerot, J. y otros. (1998). Saber y relación con el saber. Buenos Aires: Paidós.

Bion, Wilfred R. (1991): Aprendiendo de la experiencia. México: Paidós.

Blanchard Laville, C. (1996). Saber y relación con el saber. Buenos Aires: Novedades Educativas- Facultad de Filosofía y Letras (UBA).

Bleger, J. (1985). Psicohigiene y psicología institucional. Buenos Aires: Paidós

Bourdieu, P. (1986). "La ilusión biográfica", en: Actas de la Rechercher: 62-63 Traducido por Laura Castelán para la Carrera de Especialización en Formación de Formadores - UBA-.

Bourdieu, Pierre y otros (1999). La miseria del mundo. Madrid: Akal.

Bourdieu, P. (2002) Campo de poder-campo intelectual. Buenos Aires: Montressor

Bourdieu, P (1976) "Algunas propiedades de los campos". Exposición realizada en la Escuela Normal Superior (Paris). Ficha de cátedra. s/d. 1977

Bourdieu, P. (2001) Poder, Derecho y clases sociales.Bilbao: Desclee de Brower Bourdieu, P. y Wacquant, L. J. D. (1995). Respuestas. Por una antropología reflexiva. Méjico: Grijalbo.

Capra, F. (1998). La trama de la vida. Una nueva perspectiva de los sistemas vivos. Barcelona: Ed. Anagrama.

Corvalán de Mezzano, A. (1996). "Recuerdos personales-memorias institucionales: hacia una metodología de indagación histórico institucional", en: Butelman, Ida (comp.) Pensando las instituciones. Buenos Aires: Paidós.

Diaz, E. (2000). La Posciencia. El conocimiento científico en las postrimerías de la modernidad. Buenos Aires: Biblos.

Enriquez, E. (2000). Seminario de Doctorado en la Facultad de Filosofía y Letras de la UBA. Mimeo

Erikson, E. H. (1985). El ciclo vital completado. Buenos Aires: Paidós.

Etchegoyen,R(1997).Los fundamentos de la técnica psicoanalítica.Buenos Aires:Amorrortu

Fernández, L. M. (1994). Instituciones Educativas. Buenos Aires: Paidós.

- (1986). La situación de formación y sus condiciones. Una mirada pedagógica institucional al problema. Documento presentado en la Primera Reunión Técnica sobre "Requerimientos en preparación de docentes y especialistas en educación". Bogotá, Colombia. Septiembre. Mimeo.

Ferry, G. (1990). El trayecto de la formación. Los enseñantes entre la teoría y la práctica. México: Paidós.

- (1997). Pedagogía de la formación. Buenos Aires: Novedades EducativasFacultad de Filosofía y Letras (UBA).

Filloux, JC. (1996). Intersubjetividad y Formación. El retorno sobre sí mismo Buenos Aires: Novedades Educativas- Facultad de Filosofía y Letras (UBA).

Filloux, JC. (1963). La personalidad. Buenos Aires: EUDEBA.

Green, A. (1991). "Desconocimiento del inconsciente (ciencia y psicoanálisis)", en: Dorey, R. Castoriadis, C. Enriquez, E. y otros. El inconsciente y la ciencia. Buenos Aires: Amorrortur ed.

Kaës, R. y otros. (1979). Crisis, Ruptura y Superación. Análisis transicional en psicoanálisis individual y grupal. Buenos Aires: Ed. Cinco.

Kuhn, T. S. (1963). La estructura de las revoluciones científicas. Méjico, F. C. E. 
(1980). "Los paradigmas científicos", en: Barnes, Barry y otros. Estudios sobre sociología de las ciencias. Madrid: Alianza.

Mendel, G. (1996). Sociopsicoanálisis y educación. Buenos Aires: Novedades Educativas- Facultad de Filosofía y Letras (UBA).

Missenard, A. (1979). "Narcisismo y ruptura", en: Kaës, R. y otros. Crisis, Ruptura y Superación. Análisis transicional en psicoanálisis individual y grupal. Buenos Aires: Ed. Cinco.

Morin, E. (1992). El Método. Las ideas. Su hábitat, su vida, sus costumbres, su organización. Madrid: Ed. Cátedra.

--- (1993). El Método. Naturaleza de la naturaleza. Madrid: Ed. Cátedra.

Cátedra. (1994). El método. El conocimiento del conocimiento. Madrid, Ed.

Rigal, L. (2002). Notas del Seminario "Teorías Sociológicas y Formación" de la Maestría de Formación de Formadores. Facultad de Filosofía y Letras. UBA. Mimeo.

Souto, M. (1995). "La formación de formadores. Un punto de partida", en: Revista del Instituto de Investigaciones de Ciencias de la Educación. Facultad de Filosofía y Letras de la Universidad de Buenos Aires. Año IV: 7. Buenos Aires.

(1996). Reflexiones metodológicas en el proceso de investigar. Ficha de la Carrera de Formación de Formadores. Buenos Aires: Facultad de Filosofía y Letras (UBA).

Souto, M. y otros. (1999). Grupos y dispositivos de formación. Buenos Aires: Novedades Educativas- Facultad de Filosofía y Letras (UBA).

Winnicott, D. W. (1977). Realidad y juego. Buenos Aires: Granica.

\section{Notas}

\footnotetext{
i Parte de este punto ha sido publicado en Alonso, MC (2011).

ii "Uno de esos dos lugares se confería a ese otro sí-mismo cuya existencia, previa a cualquier operación, da empero lugar a su creación progresiva por la otra polaridad subjetiva, que sólo puede aprehenderse a sí misma por las operaciones reflexivas que constituyen a la precedente, y a la que designaré como repetitiva-creativa. Este dispositivo es lo que justifica el descubrimiento, a partir de allí inevitable, de transferencia como fundamento de toda operación de la psique: transferencia como desplazamiento, como proyección, como introyección, como creación, en fin, como modo de conocimiento." (Green; 1991:179).

iii La negrita es nuestra.

iv "... proceso a partir del cual el sujeto cambia (se convierte en otro) sin perder por ello su identidad, en función de influencias (que pueden, claro, percibirse a la vez como negativas y positivas) ejercidas por otro (u otros). Más generalmente: efectos más o menos recíprocos de influencias entre personas, luego entonces modificaciones." (Ardoino; 1998: 14). "Así entonces todo sujeto debe situarse en función de una bipolaridad identidad-alteración, con todos los desgarramientos, todos los descuartizamientos y los juegos diversos de las angustias y resistencias que ello supone." (Ardoino; 1998: 17).

v Noción que "corresponde más al ser del otro, a su naturaleza diferente, que a su acción propia sobre alguien". (Ardoino; 1998: 17).

vi Situación esta que en la actualidad las discusiones sobre psicoanálisis y ciencia se mantiene vigente con las particularidades del momento. En esta línea es interesante y significativo el planteo de A. Green en "Desconocimiento del inconsciente (ciencia y psicoanálisis)" donde especifica la temática del inconsciente en su relación con la ciencia, en este capítulo focaliza el momento de la creación de teoría y los obstáculos epistemológicos que tiene su origen en una no transparencia del sujeto para sí.

vii Al acercarnos al adjetivo de "clínico", vemos que éste es un modo de clarificar una práctica en la cual entran en relación: la ciencia con el hombre concreto, el hombre en situación. De esta manera el cuerpo teórico de la ciencia, la práctica que asegura la relación de la ciencia
} 
con el objeto humano y este objeto en sí mismo, conforman una tríada que resulta constitutiva a la cientificidad de las ciencias humanas.

vii "casi" está puesto intencionalmente porque habría que analizar más profundamente las razones no conscientes (implícitas simbólicas o latentes) de esa inclinación.

ix Pensamiento que se caracteriza por centrar su mirada en la relación entre conocimiento, control social y poder social referido a determinados grupos. De esta manera el curriculum es visto desde una perspectiva política porque es ahí donde se dirime dónde está el poder. (Rigal; 2002).

x Ligado al origen de clase con el cual se construye la cosmovisión del mundo. La cultura dominante contribuye a la integración real de la clase dominante (asegurando una comunicación inmediata entre todos sus miembros y distinguiéndolos de las otras clases); a la integración ficticia de la sociedad en su conjunto, así pues, a la desmovilización (falsa consciencia) de las clases dominada; a la litigación del orden, establecido por el establecimiento de distinciones (jerarquías) y la legitimación de esas distinciones. A este efecto ideológico, la cultura dominante lo produce disimulando la función de división bajo la función de comunicación: la cultura que une (medio de comunicación) es también la cultura que separa (instrumento de distinción) y que legitima las distinciones constriñendo a todas las culturas (designadas como subculturas) a definirse por su distancia por la cultura dominantes. (Bourdieu; 1977, 2000).

xi Inculcación que impone un grupo social, mecanismo principal de la reproducción social; su núcleo se encuentra en la doble naturalización como consecuencia de las inscripciones sociales en las cosas y en el cuerpo. 